\title{
A new species of Eurytoma (Hymenoptera, Chalcidoidea, Eurytomidae) from South Korea, feeding on seeds of Prunus tomentosa Thunb. (Rosaceae)
}

\author{
Duk-Young Park', Seunghwan Lee ${ }^{1,2}$
}

I Insect Biosystematics Laboratory, Department of Agricultural Biotechnology, Seoul National University, Seoul, 08826, Republic of Korea 2 Research Institute for Agricultural and Life Sciences, Seoul National University, Seoul, 08826, Republic of Korea

Corresponding author: Seunghwan Lee (seung@snu.ac.kr)

Academic editor: Petr Janšta | Received 26 February 2021 | Accepted 10 July 2021 | Published 31 August 2021

http://zoobank.org/D3E967BF-0D9D-476A-9E6D-7E696C6218C8

Citation: Park D-Y, Lee S (2021) A new species of Eurytoma (Hymenoptera, Chalcidoidea, Eurytomidae) from South Korea, feeding on seeds of Prunus tomentosa Thunb. (Rosaceae). Journal of Hymenoptera Research 85: 1-9. https://doi. org/10.3897/jhr.85.64925

\begin{abstract}
Eurytoma tomentosae sp. nov., included in the Eurytoma amygdali species-group, is described from South Korea. This species could potentially be an economically important pest, as it interferes with reproduction by attacking the seeds of the garden plant $P$. tomentosae. A key to the two Eurytoma species feeding on Prunus in South Korea is provided.
\end{abstract}

\section{Keywords}

Chalcidoidea, parasitoids, pests, Prunus tomentosa, phytophagy

\section{Introduction}

Genus Eurytoma Illiger is the largest genus constituting approximately 700 species in the family Eurytomidae. Approximately 300 species have been recorded in the Palearctic region, 100 species in the Nearctic region, 80 species in Neotropical and Oriental regions, 70 species in the Australasian region, and 45 species in the Afrotropical region. They are well known for their diverse natural histories. Most species are parasitoids of larvae of Coleoptera, Diptera, Hymenoptera, and Lepidoptera; oth- 
ers are hyperparasitoids of larvae of Ichneumonoidea (Hymenoptera) and Tachinidae (Diptera); and others are phytophagous on seeds or flesh of fruits of various plants (e.g., Asphodelaceae, Campanulaceae, Euphorbiaceae, Fabaceae, Rosaceae) (Noyes 2020).

In the Palearctic region, Lotfalizadeh et al. (2007) reported that the genus Eurytoma is polyphyletic based on phylogenetic analysis based on morphology. Additionally, they divided Eurytoma sensu stricto into 11 species-groups depending on morphological characteristics. In addition, Zerova (2010) divided them into 15 species-groups using 225 Palearctic species based on morphological characteristics. Among them, the E. amygdali species-group, which is common to the opinions of two authors (Lotfalizadeh et al. 2007 and Zerova 2010), is restricted by an antenna with a 6-segmented funicle and a one-segmented clava in the female and lack of the postgenal depression by Lotfalizadeh et al. (2007). Zerova (2010) described this species-group with a comparatively large body size (approximately $5 \mathrm{~mm}$ ), elongated body with a long metasoma, antenna with 6-segmented funicle in the female and 7-segmented funicle in the male, lower genal carina, and diameter of eyes shorter than length of gena.

The $E$. amygdali species-group is known for larvae that develop within seeds of Rosaceae. In particular, E. amygdali Enderlein, E. schreineri Schreiner, E. samsonowi Vassiliev, and E. maslovskii Nikol'skaya are pests that produce critical economic damage on cultivated trees of the genus Prunus L. (Zerova and Fursov 1991). In South Korea, E. maslovskii, first recorded by Tachikawa (1979), is a well-known pest of apricots (P. armeniaca), plums (P. mume), and peaches (P. persica). Above all, it is causing severe harm to plums every year, and even some farms that heavily damaged have suffered up to 90 percent (Lee et al. 2014).

In this study, we found an undescribed Eurytoma species associated with planted Prunus tomentosa Thunb. (Rosaceae). Larvae and pupae of the new species were discovered in seeds of host plants, and ovipositing behavior of adult females to premature fruits were observed (Fig. 2E-G). Their host, Nanking cherry (P. tomentosa), is a significant horticultural plant grown for ornamental purposes in urban areas. However, we found that seeds of the Nanking cherry can be attacked by this new Eurytoma species, resulting in an economic influence on the horticultural industry by disturbing plant reproduction. Though there have been no reports of severe damage to Nanking cherry, this new pest species requires attention. Here we describe a new species damaging $P$. tomentosa with a key to South Korean Eurytoma species associated with Prunus.

\section{Materials and methods}

Adult samples were collected by direct sweeping. Some fruits damaged by the immatures were also brought to the laboratory for rearing. Specimens are deposited at the Laboratory of Insect Biosystematics, Seoul National University (SNU). Most morphological terms follow Delvare et al. (2019).

Specimens were examined with an Olympus SZ61 stereomicroscope and photographed with a DMC 5400 digital camera attached to a Leica Z16 APO motorized 
macroscope. Serial images were combined using Zerene Stacker and digitally retouched using Adobe Photoshop CS6.

Abbreviations used in this paper as follows: SNU, Seoul National University, Seoul, South Korea; cl1-3, clava segment 1-3; F1-F6, funicular segment 1-6; MPS, multiporous plate sensilla(e); POL, the distance between posterior ocelli; LOL, distance between anterior and posterior ocellus; OOL, minimal distance between posterior ocellus and inner orbit; MPOD, maximum diameter of posterior ocellus; cc, costal cell; mv, marginal vein; pmv, postmarginal vein; stv, stigmal vein; Gt1-Gtx, gaster tergites 1 to x.

\section{Taxonomy}

\section{Key to South Korean species of Eurytoma associated with Prunus}

Antenna with F1-6 cylindrical; with setae as long as half-length of funicular segment (Figs 1B, 3B); petiole of metasoma wider than long (Fig. 1G)

female 2

- $\quad$ Antenna with F1-5 or 6 petiolate; with setae as long as length of funicular segment (Figs 2B, 3F); petiole of metasoma at least $2 \times$ longer than wide (Figs 2D, 3H) male 3

2 Head with temples $0.4 \times$ as long as length of eye in dorsal view (Fig. 3C); first funicular approximately $3 \times$ as long as wide, F2-6 about $2 \times$ longer than wide (Fig. 3B); OOL 2.5-2.8x as long as MPOD (Fig. 3C); propodeum without straight carina medially; metasoma $1.16-1.17 \times$ as long as head+mesosoma, syntergum as long as Gt6 and upturned; body length more than $6 \mathrm{~mm}$. On apricots (P. armeniaca), plums (P. mume), and peaches (P. persica)

\section{Eurytoma maslovskii Nikol'skaya}

- $\quad$ Head with temples $0.32-0.33 \times$ length of eye in dorsal view (Fig. 1D); F1 approximately $1.5 \times$ as long as wide, F2 -6 about $1.1 \times$ longer than wide (Fig. 1B); OOL 2.0× as long as MPOD (Fig. 1D); propodeum with distinctly straight carina anterior margin to middle of propodeum (Fig. 1G); metasoma $0.90-0.93 \times$ as long as head+mesosoma; syntergum as half as length of Gt6 and not upturned; body length less than $5 \mathrm{~mm}$. On Nanking cherry (P. tomentosa)

Eurytoma tomentosae sp. nov.

3 Head with temples $0.55-0.56 \times$ length of eye in dorsal view (Fig. 1D); antenna with F1-5 petiolate; OOL 3.1× as long as MPOD (Fig. 3G); propodeum without straight carina medially, petiole more carinate (Fig. $3 \mathrm{H})$; body length more than $5 \mathrm{~mm}$. On apricots (P. armeniaca), plums (P. mume), and peaches (P. persica)

Eurytoma maslovskii Nikol'skaya

- $\quad$ Head with temples $0.32-0.33 \times$ length of eye in dorsal view (Fig. 1D); antenna with F1-6 petiolate; OOL 2.0-2.3x as long as MPOD (Fig. 2C); propodeum with distinctly straight carina medially, petiole less carinate to coriaceous (Fig. 2D); body length more than $4 \mathrm{~mm}$. On Nanking cherry (P. tomentosa) 


\section{Eurytoma tomentosae sp. nov.}

http://zoobank.org/178DA8C6-4A0B-49C3-9F05-0C666838B14E Figs $1 \mathrm{~A}-\mathrm{H}, 2 \mathrm{~A}-\mathrm{G}$

Type material. Holotype female; South Korea: Incheon, Yeonsu-gu, Solsaem-ro 43beon-gil, 42, Mt. Cheongnyang, 37²5'20.6"N, 126³9'50.7"E, 12.v.2019, found on fruits of Prunus tomentosa, Duk-Young Park (in SNU). Paratype. 7 females, 10 males, same data as holotype, 5 females, 16 males (in SNU); same data as holotype except 11.v.2019, Jihwan Park (in SNU).

Etymology. The species is named after the host plant, Prunus tomentosa.

Diagnosis. This new species differs from others of amygdali species-group in the comparatively small body length, short funicular segments, metasoma shorter than head+mesosoma, especially the syntergum half as long as Gt6 and not upturned.

Description. Female (Fig. 1A, habitus). Body length $4.61-4.74 \mathrm{~mm}$, including ovipositor. Antenna (Fig. 1B) black except radicle and clava dark brown; scape sparsely setose, spindle-like. Body black except mandible reddish-brown to dark apically, labial and maxillary palpi (Fig. 1C) black basally to apical tips yellowish-brown, knees brown to whitish-yellow medially, tibiae narrowly apically brown, ovipositor sheaths pale; with hair-like to slightly lanceolate yellow setae. Fore wing (Fig. 1H) pale yellowish, but deeply yellow infuscated below apical half of submarginal vein to stigmal vein; veins brownish-yellow and setae yellow. Hind wing yellowish-hyaline with pale-yellow setae.

Head 1.87-1.97x as wide as long and temples as long as one third of eye length in dorsal view (Fig. 1D); 1.39-1.52x as wide as high in frontal view (Fig. 1C); OOL:POL:LOL:MPOD = 2.0:2.5-2.6:1.0-1.1:1.0; scrobal depression adjoined from anterior ocellus, somewhat strigulate, carinate laterally and ground-shaped bottle in form; frons with yellowish lanceolate setae except between eyes and ocelli. Malar space $0.70 \times$ as long as height of eye. Mandible 3-toothed. Vertex and upper face entirely areolate except between eyes and ocelli coriaceous-punctured; lower face areolate-strigose converged towards clypeus, but clypeus smooth to somewhat strigose; malar sulcus shallowly and widely groved. Lateral outline of gena distinctly convex in frontal view; genal carina present.

Antenna (Fig. 1B). Scape slightly swollen anteromedially; 3.67-3.75x as long as wide. Pedicel short, $0.9-0.95 \times$ as long as wide. Anellus $0.6-0.66 \times$ as long as wide. Funicle 6-segmented; F1 approximately $1.5 \times$ as long as wide; F2-F6 slightly longer than wide; each funicular with two rows of MPS; all setae subdecumbent. Clava 1-segmented; $2.87-2.99 \times$ as long as wide.

Mesosoma (Fig. 1E, F). In dorsal view, mesosoma 1.60-1.61× as long as wide; pronotum, and mesoscutum respectively $2.22 \times$ and $1.5 \times$ as wide as long; mesoscutellum $1.11 \times$ as long as wide. Propodeum approximately $125^{\circ}$ angle to the plane of scutellum. Pro- and mesonotum densely coriaceous-punctured except anterior area of mesoscutal lateral lobe smooth in dorsal view and neck alutaceous. Sides of pronotum straight, not convex. Notauli distinctly impressed, narrow. Axilla clearly separated from scutellum by impressed axillar groove. Propodeum with irregularly rugulose-areolate and concave mesal area bearing distinct median carina on anterior half, lateral area areolate (Fig. 1G). Prepectus smooth except medial area to anterior and anteroventral margin rugulose. 

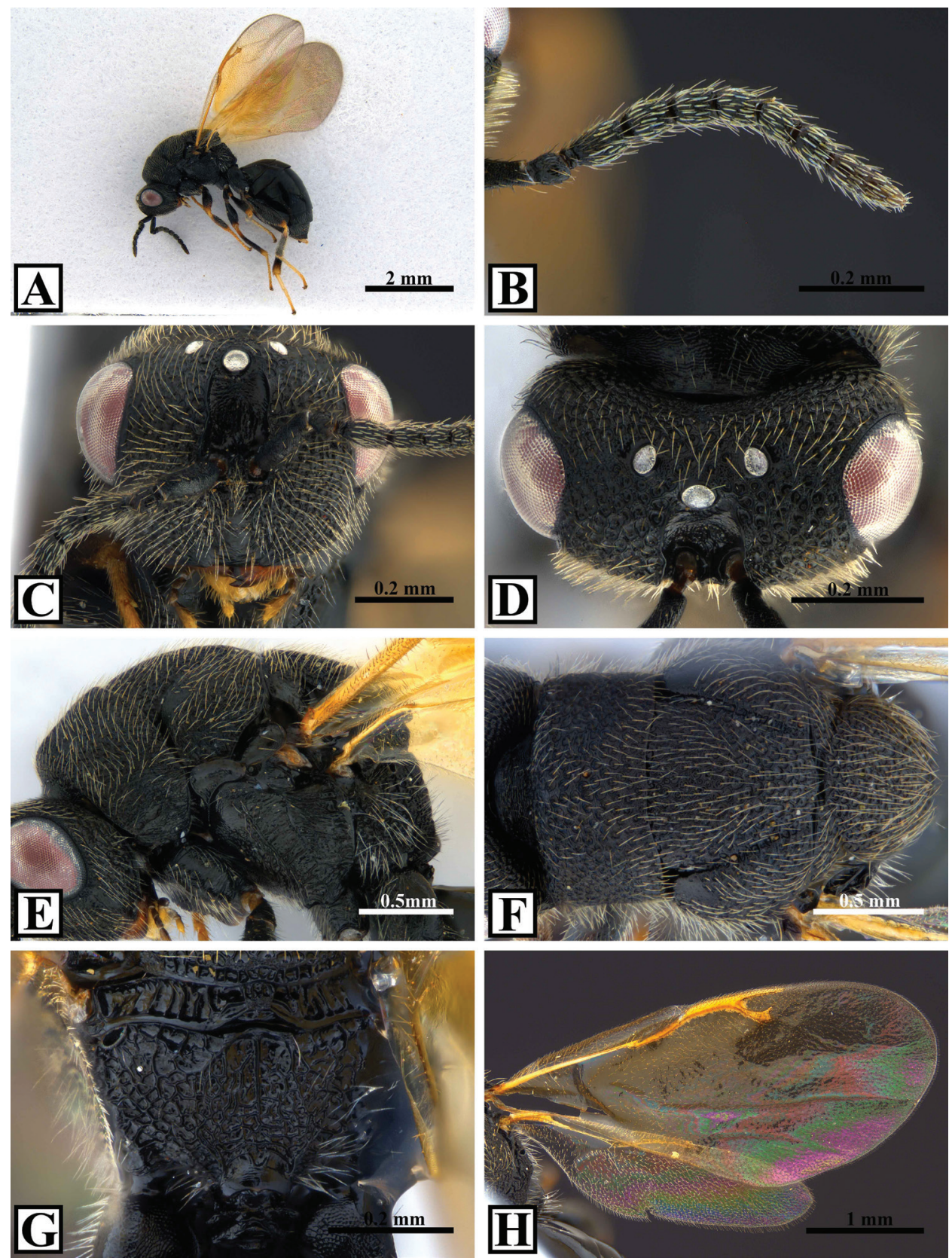

Figure I. A-H Eurytoma tomentosae, female A habitus B antenna $\mathbf{C}$ head, frontal view $\mathbf{D}$ head, dorsal view $\mathbf{E}$ mesosoma, lateral view $\mathbf{F}$ mesosoma, dorsal view $\mathbf{G}$ propodeum $\mathbf{H}$ wing.

Tegula entirely smooth except posterior and ventral margin imbricate; with 2 distinct setae. Epicnemium hardly margined laterally by low epicnemial carina. Mesosternal shelf absent. Mesepisternum with variously smooth to strigose upper one-fourth and confused-areolate in lower three-fourth; adscrobal carina delimiting anteriorly femoral 

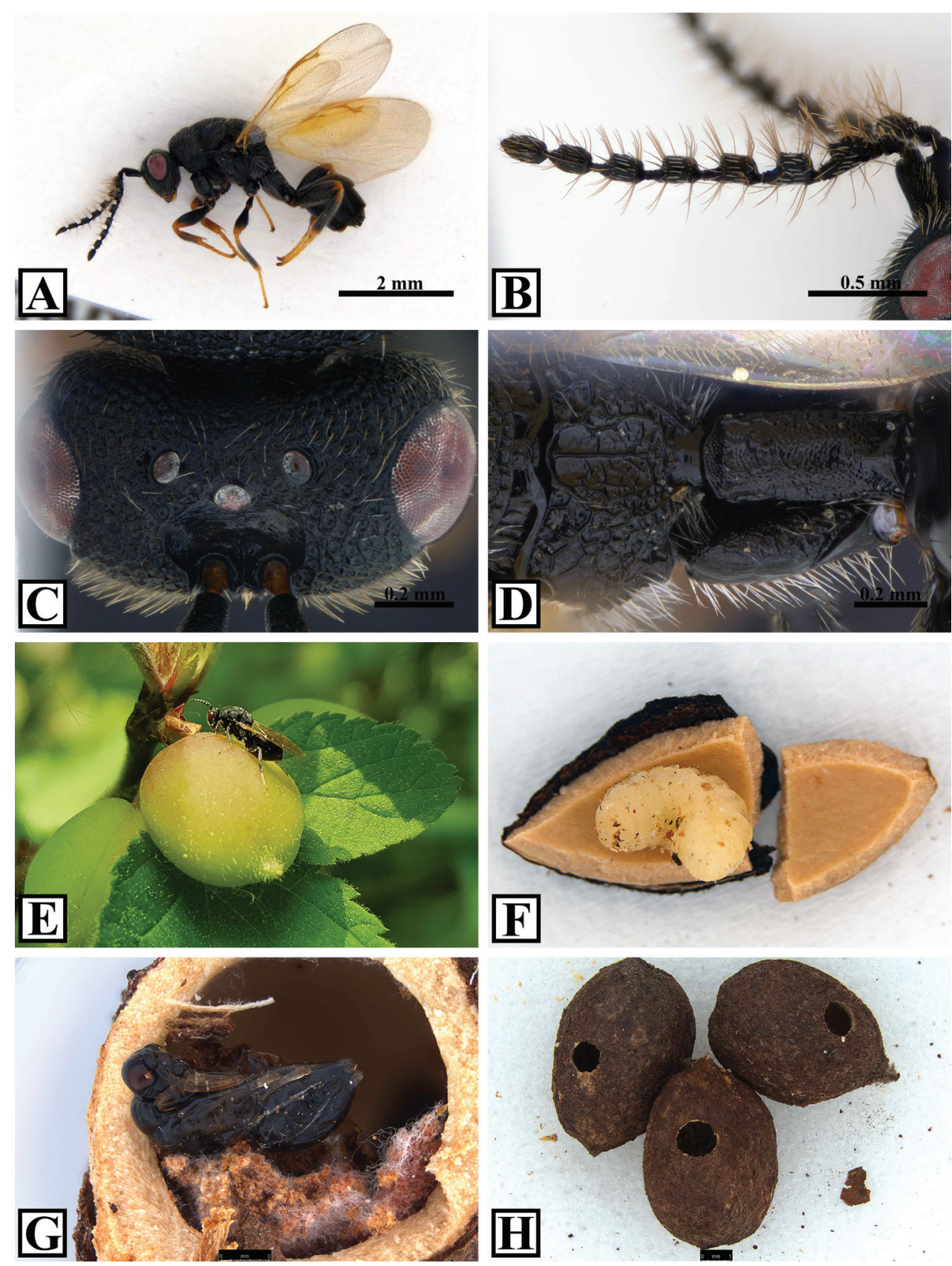

Figure 2. A-H Eurytoma tomentosae $\mathbf{A}-\mathbf{D}$ male $\mathbf{A}$ habitus $\mathbf{B}$ antenna $\mathbf{C}$ head, dorsal view $\mathbf{D}$ propodeum and petiole $\mathbf{E}$ ovipositing behavior of female $\mathbf{F}$ larva in seed $\mathbf{G}$ pupa in seed $\mathbf{H}$ seed of $P$. tomentosa after adults of $E$. tomentosae emerged. 


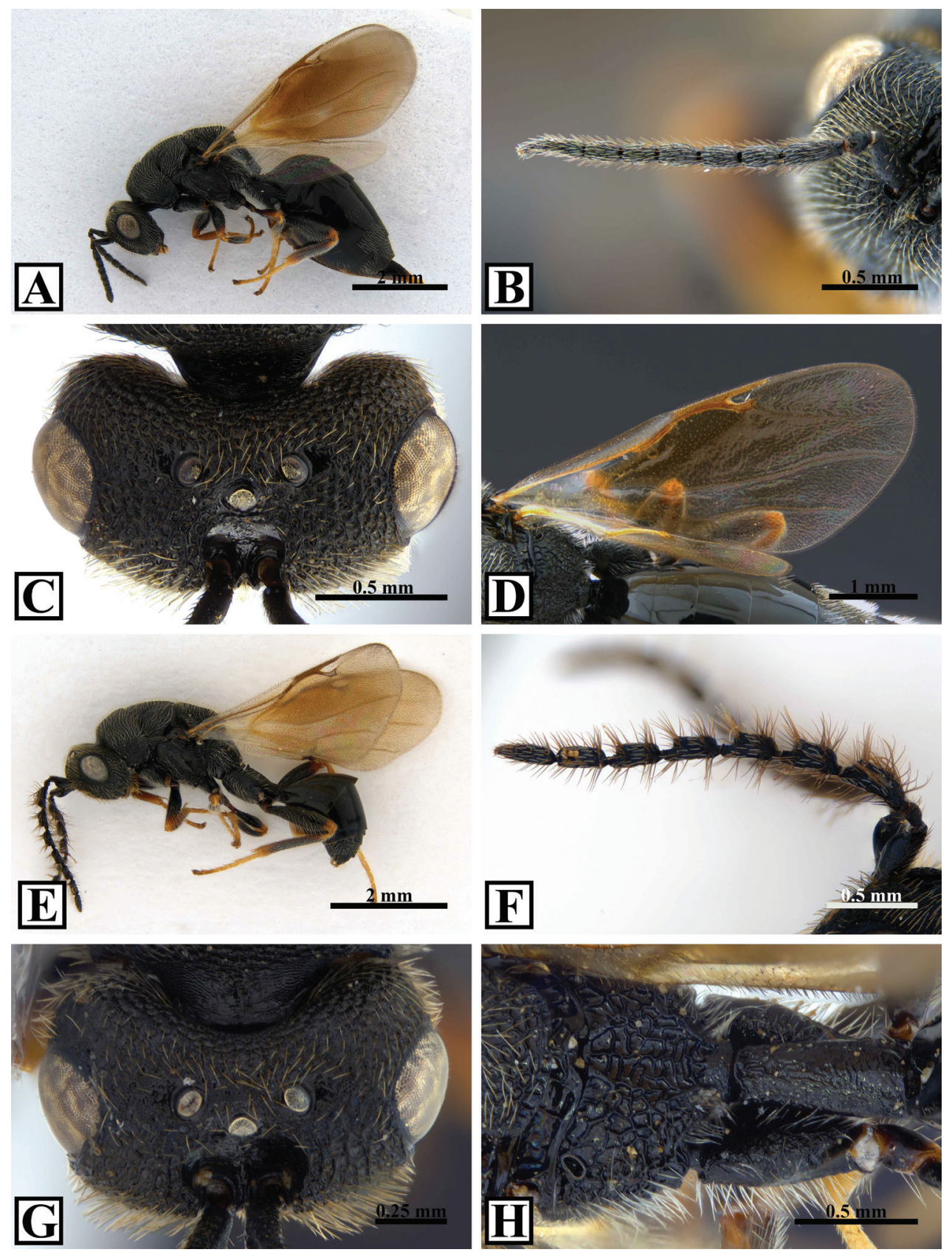

Figure 3. A-D Eurytoma maslovskii female $\mathbf{A}$ habitus $\mathbf{B}$ antenna $\mathbf{C}$ head, dorsal $\mathbf{D}$ wing $\mathbf{E}-\mathbf{H}$ E.maslovskii male $\mathbf{E}$ habitus $\mathbf{F}$ antenna $\mathbf{G}$ head, dorsal $\mathbf{H}$ propodeum to petiole. 
depression and reaching ventrally the mesocoxal foramen; femoral depression and mesepimeron with irregular carinulae, but the latter with smooth posterior margin. Metepimeron areolate and bearing long and thin erect setae, merging to propodeum posteriorly. Legs. Procoxa imbricate except smooth medially, without carina; setation bare medially. Mesocoxa without lamella. Metacoxa bare dorsally at base. Fore wing (Fig. 1H) 2.34-2.39 $\times$ as long as wide, cc: mv: pmv: stv $=5.3-5.5: 1.3-1.4: 1.2-1.4: 1.0$. Stigmal vein approximately an angle of $35^{\circ}$ with the postmarginal vein. Stigma with sharp uncus.

Metasoma. Petiole wider than long, with slightly rough-coriaceous dorsal surface and highly rised carina transversally. Gaster ovate and smooth, about $1.59-1.74 \times$ as long as length and 1.75-1.82× as long as width. Gt4 slightly longer than Gt3; with short setae on anterior admarginal area. Gt5 and Gt6 similar in length; with hair-like yellow setae. Syntergum short and not upturned. Apex of ovipositor sheath round.

Male (habitus Fig. 2A). Body length 3.99-4.0 mm. Morphologically similar to females except the following. Antenna (Fig. 2B) with funicle 6-segmented and clava 1-segmented; funicular segments petiolate, the bodies almost square. Head (Fig. 2C) with OOL: POL: LOL: MPOD = 2.0-2.3:2.8:1.2:1.0. Propodeum (Fig. 2D) less ruguloseareolate than female. Metasoma with long petiole punctured and less rugulose (Fig. 2D).

Host. Prunus tomentosa Thunb. (Rosaceae).

Biology. Adults of this species were observed emerging from seeds and mating in May. After mating, females oviposit inside premature fruits, and hatching larvae develop by eating the insides of seeds. They overwinter in a larval stage and pupate in spring before escaping from the seeds.

Distribution. South Korea.

\section{Discussion}

Eurytoma tomentosae sp. nov., is included in the E. amygdali species-group according to the definition provided by Lotfalizadeh et al. (2007) and Zerova (2010) based on a comparatively large body size (approximately $5 \mathrm{~mm}$ ), antenna with 6-segmented funicle in the female and 7-segmented funicle in the male, lack of postgenal depression, lower genal carina.

The new species would be keyed out as E. schreineri using the key of Zerova and Fursov (1991) and Zerova (2010), because the metasoma is slightly longer than the mesosoma, the marginal vein is longer than the stigmal vein, and the syntergum is not upturned. However, it is distinguished from E. schreineri in having the metasoma shorter than the head plus mesosoma, the syntergum half as long as Gt6, and all funiculars with comparatively long setation in males. In addition, this species might be confused with the geographically close species E. maslovskii, distributed in Far East regions (Shandong, China; Japan; Korea; Primor'ye Kray, Russia) (Noyes 2020). However, E. tomentosae differs from E. maslovskii in having a distinctly shorter body length, less than $5 \mathrm{~mm}$ in the female and at most $4 \mathrm{~mm}$ in the male, comparatively short funicular segments in both sexes, OOL at most $2.0 \times$ (female) 
and 2.0-2.3× (male) as long as MPOD, propodeum with distinctly straight carina, metasoma at most slightly longer than mesosoma, and syntergum not upturned and half as long as Gt6.

\section{Acknowledgements}

We thank Jihwan Park for giving us the information on collecting the location of specimens. This work was supported by a grant from the National Institute of Biological Resources (NIBR) funded by the Ministry of Environment (MOE) of the Republic of Korea (NIBR202002205 and NIBR202130203), by Basic Science Research Program through the National Research Foundation of Korea (NRF) funded by the Ministry of Education (NRF-2020R1I1A2069484), and by Survey and identification of insect pests of small area-cultivated crops in central areas of Korean peninsula (Project Code PJ01450704) supported by Rural Development Administration, South Korea.

\section{References}

Delvare G, Ribes Escolà A, Stojanova AM, Benoit L, Lecomte J, Askew RR (2019) Exploring insect biodiversity: the parasitic Hymenoptera, chiefly Chalcidoidea, associated with seeds of asphodels (Xanthorrhoeaceae), with the description of nine new species belonging to Eurytomidae and Torymidae. Zootaxa 4597(1): 001-090. https://doi.org/10.11646/ zootaxa.4597.1.1

Lee SM, Kim SJ, Yang CY, Shin JS, Hong KJ (2014) Host Plant, Occurrence, and Oviposition of the Eurytomid wasp Eurytoma maslovskii in Korea. Korean Journal of Applied Entomology 53(4): 381-389. https://doi.org/10.5656/KSAE.2014.10.0.047

Lotfalizadeh H, Delvare G, Rasplus J-Y (2007) Phylogenetic analysis of Eurytominae (Chalcidoidea: Eurytomidae) based on morphological characters. Zoological Journal of the Linnean Society 151: 441-510. https://doi.org/10.1111/j.1096-3642.2007.00308.x

Noyes JS (2020) Universal Chalcidoidea Database. World Wide Web electronic publication. http://www.nhm.ac.uk/chalcidoids

Tachikawa (1979) Eurytoma marlovskii Nikolskaja newly discovered from Korea (Hymenoptera: Chalcidoidea - Eurytomidae). Transactions of the Shikoku Entomological Society 14(3-4): 181-183.

Zerova MD (2010) Palaearctic species of the genus Eurytoma (Hymenoptera, Chalcidoidea, Eurytomidae): morphological and biological peculiarities, trophicalassocations and key to determination [In Russian]. Vestnik Zoologii, Kiev 24, 203 pp.

Zerova MD, Fursov VN (1991) The Palaearctic species of Eurytoma (Hymenoptera: Eurytomidae) developing in stone fruits (Rosaceae: Prunoideae). Bulletin of Entomological Research 81(2): 209-219. https://doi.org/10.1017/S0007485300051294 